\section{The meeting with Dr Cameron}

\author{
R. J. Tayler
}

Cosmogonical Processes. Edited by W. D. Arnett, C. J. Hansen, J. W. Truran and S. Tsuruta. VNU Science Press: 1986. Pp. 300. DM 135.

A. G. W. (Al) Cameron has made many notable contributions to astrophysics in the past 30 years. He was trained as a nuclear physicist, but a latent interest in astrophysics was activated by Paul Merrill's 1952 discovery of the unstable element technetium in stars. Cameron started to work on nuclear astrophysics and in 1957, at about the same time as Burbidge, Burbidge, Fowler and Hoyle published their celebrated paper on stellar nucleosynthesis in Reviews of Modern Physics, he wrote a Chalk River report which identified the crucial processes in the formation of the elements. $\mathrm{He}$ also stressed the probable importance of neutron stars and, with students and colleagues, calculated their properties before the discovery of pulsars caused most astronomers to believe in their existence.

In March 1985 four of Cameron's former students organized a symposium at Boulder, Colorado, to celebrate his sixtieth birthday, and this volume contains written versions of all but one of the talks together with an autobiographical sketch by Cameron himself. The book contains review articles on a variety of topics of current astronomical interest, most of them being ones to which Cameron has made significant contributions. The articles are generally written in a manner which makes them accessible to readers who are not experts in the fields concerned, and the book is one of the better examples of published conference proceedings.

One recurring theme concerns the questions of what the Universe is made of and how much of it there is. There is a theoretical prejudice that the Universe should have a critical density such that it will just expand for ever. In this case, much of the matter is probably composed of nonbaryonic elementary particles of a type whose existence has not yet been confirmed. Press describes how such particles might accumulate in the centre of the Sun, and might then provide a solution to the long-lasting solar neutrino problem, Rees discusses how galaxy formation might have been affected by this non-luminous matter, and Fowler asks whether the age of the Universe can be compatible with the ages of objects in it if the Universe does have critical density.

The origin of the chemical elements,

Cameron's early interest, is the subject of several contributions. Pagel reviews the chemical evolution of galaxies and explains how the observed variation of chemical composition with position and with stellar age may be explained by infall of unprocessed gas, by outflow of processed material or by a variation with time of the stellar mass function (or, more likely, by a combination of all three). Clayton discusses the peculiar isotopic abundances which are found in some meteorites and Hillebrandt's contribution on nucleosynthesis in supernovae indicates that, while substantial progress has been made since 1957, much remains to be understood.

The remaining chapters cover other topics in which Cameron has had a particular interest: supernovae and neutron

\section{Advice in analysis}

\section{Rodger Staden}

Software Directory for Molecular Biologists: A Complete Guide to the Selection of Computer Software for the Management and Analysis of Molecular Sequences. By Christopher J. Rawlings. Macmillan, London/Stockton, New York: 1986. Pp. 412 . £40, $\$ 80$.

IT Is ten years since the publication of the articles that described the rapid and simple methods used to determine sequences of DNA. So efficient are these techniques that it has been necessary to create international data libraries to record the results. These collections have grown so that the European library now contains over eight million nucleotides, and the record for the longest individual sequence has tripled every two or three years.

Computers are used to help determine and analyse all this genetic data, but whereas the sequencing techniques were picked up fairly quickly, the spread of computer programs for handling the data was hampered by several problems. The users of the software were computernaive, and lacked suitable hardware; programs were not easily run on different machines, and it was hard to find people to convert them for local facilities. By modern standards the early programs were not user-friendly, and the concurrent emergence of personal computers showed what could be done. These factors, combined with the decision of Nucleic Acids Research, the journal that publishes most of the work in this area, not to referee formally papers submitted for its special computer issues, have led to a proliferation of software for analysing sequences. So we have an area where computers are essential, where users are computer-naive and the software is abundant but of stars and the origin of cosmic rays, and the formation of stars and of the Solar System. Modestly, the four organizers of the meeting chose not to present any papers or to write any articles for the book. However, anyone familiar with the field will know that they have all made major contributions to the subject and that Cameron's contribution to astronomy through the training and inspiration of research students has been as important as his own distinguished research. This is the clear message of the book, which is alike a worthy tribute to Cameron and a very useful summary of the present state of research in the areas discussed.

R. J. Tayler is a Professor in the Astronomy Centre, University of Sussex, Brighton BNI $9 Q H, U K$ unknown usefulness, and thus an obvious need for a source of information on the subject. This book aims to fill the vacant niche.

Its nomenclature and glossary cover the field, and explain the relevant computer terminology including that used in the software employed for sequence analysis; it outlines the use of word-processing packages, data-management systems, terminal emulation and even spreadsheets. More than 75 per cent of the contents is taken up by the well-cross-referenced directory of software, with 60 per cent of the references being from the special computer issues of Nucleic Acids Research.

For those knowing the field, the book will be very helpful as a single source of reference. But for its intended audience of newcomers wishing to choose software and hardware, although useful, it is weak on two important points. First, it does not address the problem of unrefereed publications, as no real opinions are given about the value of the packages described. Secondly, this is a fast-moving area, and a book - which is inevitably already out of date, and in which I could find no mention of future updates - is possibly an inappropriate vehicle for much of the information provided.

Rodger Staden is at the Medical Research Council Laboratory of Molecular Biology, Hills Road, Cambridge CB2 $2 Q H, U K$.

\section{Computing applied}

Other recent books on the use of computers in science:

- Chemistry by Computer: An Overview of the Applications of Computers in Chemistry by Stephen Wilson (Plenum; \$37.50).

- Computer Software Applications in Chemistry by Peter C. Jurs (Wiley; $\$ 34.95, £ 33.75$ ).

- A second edition of Microcomputers and Physiological Simulation by James E. Randall (Raven; \$34).

- Scientific and Engineering Applications with Personal Computers by Raymond Annino and Richard Driver (Wiley; $\$ 47.50, £ 45.65$ ). 